\title{
Community hospitals: an under-recognized resource for palliative care
}

\author{
Sheila Payne $\mathrm{PhD}^{1} \quad$ Chris Kerr $\mathrm{PhD}^{2}$ Sheila Hawker $\mathrm{PhD}^{2}$ David Seamark $\mathrm{PhD}^{3}$ Carol Davis $\mathrm{FRCP}^{4}$ \\ Helen Roberts FRCP ${ }^{4}$ Nicola Jarrett PhD $^{5}$ Paul Roderick FFPHM ${ }^{6}$ Helen Smith DM $^{7}$
}

J R Soc Med 2004;97:428-431

SUMMARY

In the UK there are concerns that, in certain groups of dying patients such as the old, those with non-cancer diagnoses and those in rural areas, the quality of care is unacceptably variable. There has been no systematic survey of the extent to which community hospitals provide general palliative care for such patients. Therefore, by means of a structured questionnaire we asked senior nurses/managers at all $\mathbf{4 7 8}$ community hospitals in the UK for information on staff expertise, facilities and specialist equipment, liaison arrangements with specialist palliative care providers, priorities, practice and policy in end-of-life care.

Of the 346 hospitals (72\%) that responded, only 28 were in urban areas. $73 \%$ of hospitals employed at least one nurse with additional training in palliative care, $72 \%$ had access to 24 -hour specialist palliative care advice and $51 \%$ had separate overnight accommodation for relatives, but only $22 \%$ had designated palliative care beds. Most hospitals did not have written policies or guidelines for patient assessment or symptom control.

These findings add to evidence that community hospitals represent an important resource to improve access to palliative care for groups that are currently under-served.

\section{INTRODUCTION}

Within the past decade there has been growing recognition that general practitioners, community nurses and other primary-care-based professionals provide the mainstay of support to patients who are nearing the end of life and to their families. ${ }^{1-4}$ General practitioners regard care for dying patients as important, rewarding and satisfying and see themselves as coordinators of care. ${ }^{5}$ Fordham and colleagues ${ }^{6}$ went further by arguing that palliative medicine is but a specialized version of general practice. This proposal sparked considerable debate. ${ }^{7-9}$ New initiatives in primary care have sought to raise the standard of general palliative care by implementing agreed multidisciplinary protocols, ${ }^{10}$ and the National Institute for Clinical Excellence (NICE) has made specific recommendations about the delivery of general palliative care by primary care practitioners. $^{11}$

${ }^{1}$ Palliative and End-of-Life Care Research Group, University of Sheffield, Bartolome House, Winter Street, Sheffield S3 7ND; ${ }^{2}$ Social Work Studies, University of Southampton; ${ }^{3}$ The Honiton Group Practice, Honiton, Devon; ${ }^{4}$ Elderly Care Research Unit, Southampton General Hospital; ${ }^{5}$ School of Nursing and Midwifery, University of Southampton; ${ }^{6} \mathrm{Health}$ Care Research Unit, Southampton General Hospital; ${ }^{7}$ Division of Primary Care and Public Health, Brighton and Sussex Medical School, Brighton, UK

Correspondence to: Professor S Payne
When asked where they would wish to receive terminal care and die, most patients express a preference for home, but often this is impossible because suitable carers are lacking, the symptoms are complex and hard to manage or housing conditions are wrong. ${ }^{12}$ General practitioners use community hospital beds to maintain continuity, but changes in their out-of-hours responsibilities have increased the likelihood of a patient being admitted to an acute hospital. ${ }^{13,14}$ Hospices and specialist palliative care services are a scarce resource and closely linked with cancer. ${ }^{15}$ Concerns have been raised about the inequity of access to specialist palliative care services-particularly for older patients, black and ethnic minority groups, those with noncancer diagnoses and those living in rural areas. ${ }^{16}$ Moreover, the specialist services may be poorly geared to the needs of old people, in whom dying tends to have a longer trajectory than in younger patients. ${ }^{17}$

Community hospitals offer a range of services with an emphasis on care for older people and could be regarded as appropriate places for terminal care-readily accessible to family and friends, staffed by local people and allowing continuing care by general practitioners. ${ }^{18}$ However, concerns have been identified about low staffing levels, unsatisfactory standards of care and poor organization. ${ }^{19}$ When Seamark and co-workers compared the care given to dying cancer patients in twelve community hospitals with 
that in a hospice they found little difference in quality, ${ }^{20,21}$ but in the community hospitals they did identify instances of inadequate nursing provision, suboptimal bereavement care and poor communication. If healthcare planning is to incorporate community hospitals as places for the delivery of palliative care, it is important to know what resources they can offer. We therefore surveyed community hospitals in the UK to determine their resources and facilities for general palliative care.

\section{METHODS}

A structured questionnaire was developed in consultation with the Community Hospitals Association and pilot tested in two stages with senior nurses and/or managers from 13 community hospitals to establish face validity, relevance and feasibility and to reduce item ambiguity. The subject matter included staff expertise in palliative care, facilities and specialist equipment, liaison arrangements with specialist palliative care providers, priorities, practice and policy in end-of-life care. Pilot testing prompted minor modifications to the wording.

All 478 community hospitals identified on the Community Hospitals Association database for the UK in 2001 were then invited to participate in the survey, and non-responders received two reminder letters. The senior nurse/manager in each was invited to return the completed questionnaire by mail or to answer the same questions by telephone. The mailed and telephoned data were combined in a single database and analysed by use of SPSS 11.0 for Windows. Hospitals were categorized by number of beds according to published recommendations - small $<30$, medium-sized $30-49$, large 50 or more. ${ }^{22}$ Numerical responses were assessed by descriptive statistics and crosstabulations; open textual responses were coded by means of content analysis.

\section{RESULTS}

$346(72 \%)$ of the 478 community hospitals responded. Figure 1 shows the recruitment flow. Comparison of respondents with non-respondents revealed no systematic bias in terms of geographical location or size of hospital. However, hospitals where there was shared care between general practitioners and consultants were significantly less likely to respond $\left(\chi^{2}=13.85, \mathrm{df}=2 ; P=0.001\right)$ than those led by one or the other.

Community hospitals were located throughout the UK-England 324, Scotland 85, Wales 58, Northern Ireland 4. Most were in rural and suburban areas $(n=214)$ or coastal areas within $8 \mathrm{~km}$ of the sea $(n=104)$, with a minority in urban areas $(n=28)$. The median number of beds was 26 (range 6-178): 199 had less than 30, 99 had 30-49 and 48 had 50 or more. Only 78 (22\%) hospitals reported

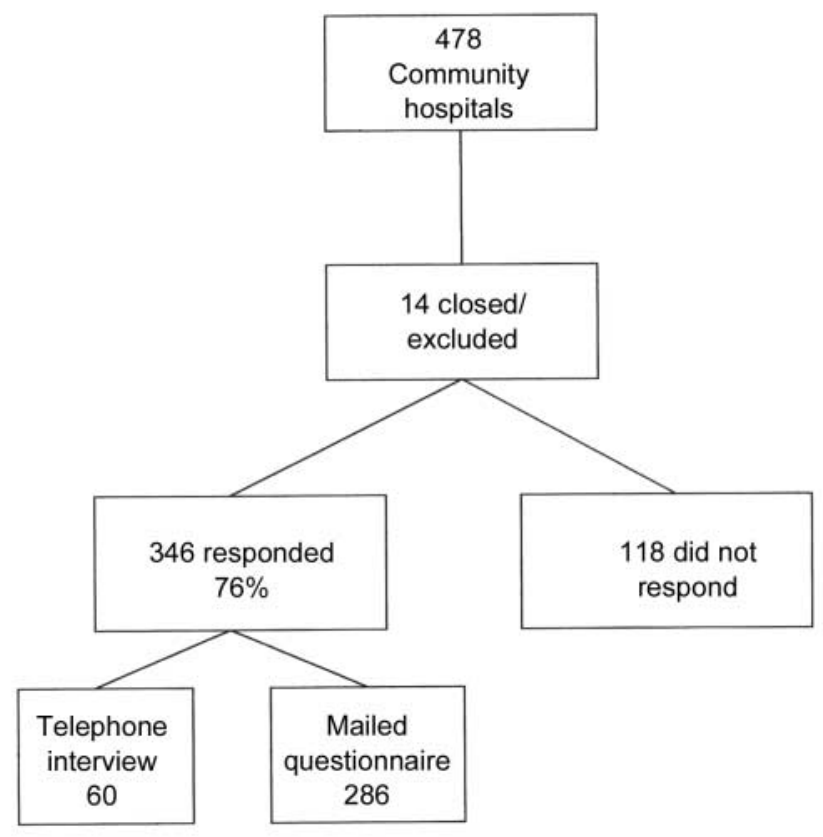

Figure 1 Community hospitals recruitment flow chart

having designated palliative care beds (median 2, range 1-6) and 176 (51\%) reported having separate overnight accommodation for patients' relatives. Management of the beds was as follows: general practitioner 149 hospitals; consultant 35; shared care 161; nurse-led 1. In 208 hospitals $(60 \%)$ at least one of the doctors with admitting rights (general practitioner or consultant) had a special interest in palliative care, while 251 (73\%) employed one or more nurses (median 2, range 1-14) with postregistration training in palliative care. Continuing education in topics relevant to palliative care was provided for staff by 318 hospitals (92\%).

Table 1 summarizes responses to questions about access to, and liaison arrangements with, specialist palliative care providers. The median distance to the nearest hospice was $24 \mathrm{~km}$, though for two hospitals on Scottish islands the distance was more than $300 \mathrm{~km}$. Virtually all hospitals had specialist equipment such as syringe drivers and pressure reducing beds.

$315(91 \%)$ hospitals were visited routinely and regularly by Christian ministers but only 2 by ministers of other faiths. $174(50 \%)$ employed a staff member trained in complementary therapies. The proportions of hospitals receiving visits from a range of professional staff was as follows: physiotherapists $93 \%$, occupational therapists $86 \%$, pharmacists $75 \%$, social workers $63 \%$, counsellors $17 \%$. Only a minority of hospitals had written policies or guidelines specific to the following aspects of palliative care: symptom control $41 \%$, bereavement support $25 \%$, carer needs assessment $21 \%$, non-cancer diagnoses $21 \%$, black and ethnic minority patients $13 \%$. 
Table 1 Resources for providing palliative care in community hospitals located in rural, urban and coastal areas of the UK

\begin{tabular}{|c|c|c|c|c|}
\hline Resource & $\begin{array}{l}\text { Rural/suburban } \\
\text { hospitals } \\
(n=214)\end{array}$ & $\begin{array}{l}\text { Urban } \\
\text { hospitals } \\
(\mathrm{n}=28)\end{array}$ & $\begin{array}{l}\text { Coastal } \\
\text { hospitals } \\
(n=104)\end{array}$ & $\begin{array}{l}\text { All hospitals } \\
(\mathrm{n}=346)\end{array}$ \\
\hline Median bed number & 25 & 28 & 26 & 26 \\
\hline Median distance to hospice (km) & 24 & 10 & 33 & 24 \\
\hline$\%$ Employing nurses with training in palliative care & $76 \%$ & $57 \%$ & $70 \%$ & $73 \%$ \\
\hline$\%$ Access to $24 \mathrm{~h}$ SPC advice & $75 \%$ & $68 \%$ & $68 \%$ & $72 \%$ \\
\hline $\begin{array}{l}\% \text { With regular routine visits from consultant in palliative } \\
\text { medicine }\end{array}$ & $11 \%$ & $18 \%$ & $9 \%$ & $11 \%$ \\
\hline$\%$ With regular routine visits from SPC nurse & $44 \%$ & $18 \%$ & $43 \%$ & $42 \%$ \\
\hline
\end{tabular}

$\mathrm{SPC}=$ Specialist palliative care; rural/suburban=villages, small towns and outskirts of larger towns; coastal=within $8 \mathrm{~km}$ of coast

\section{DISCUSSION}

This survey, yielding information from across the UK, indicates that community hospitals represent an important resource for general palliative care, and might be developed to supply the unmet needs of certain groups, especially in rural areas. For patients requiring inpatient admission for general rather than specialist palliative care, community hospitals may offer an attractive alternative to the scarce and expensive hospice or the acute general hospital. However, there is room for improvement. Few of the community hospitals reported having written policies on topics related to palliative care to guide staff; whilst there is conflicting evidence about their practical value, clinical guidelines do provide a useful benchmark for clinical governance and audit. ${ }^{23,24}$ Only half had separate overnight facilities for patients' relatives - whereas hospices nearly always do. Few hospitals were able to keep a room specifically for patients needing palliative care, probably because of competing pressures, and this may mean that dying patients sometimes have to be cared for in less than optimal surroundings.

Certain limitations of the study must be acknowledged. The survey data were collected by two methods, mailed questionnaires and structured telephone interviews, and it may be that these elicited somewhat different responses (they were not analysed separately); for example, telephone interviews allowed for greater clarification of questions or terminology. However, the questions were the same. One must also be cautious about generalizing from a survey with a response rate of only $72 \%$, though on comparison with the original sample we found no biases in terms of location or size of hospital. More importantly, because these are self-report data, we are unable to comment on the quality of care provided.

Because previous research has tended to group all hospitals together, we know little about how patients and their relatives regard end-of-life care in community care, these should be distinguished from larger establishments such as district general hospitals. On existing evidence, community hospitals see palliative care as part of their work, and a qualitative study in Scotland indicated that general practitioners feel confident admitting terminally ill older patients to such hospitals. ${ }^{26}$ With the greater recognition of the special palliative care needs of older patients and those with diagnoses other than cancer, ${ }^{16}$ and the continuing debate about different models of specialist palliative care ${ }^{13}$ community hospitals offer advantages including continuity of care from general practitioners, ready access for family members and seemingly good links with palliative care professionals, especially Macmillan nurses. The National Service Frameworks for Older People $^{27}$ and Cardiovascular Disease ${ }^{28}$ highlight the importance of improving the quality of care for all dying people and facilitating their end-of-life choices. In the context of improving cancer care, the Supportive and Palliative Care Guidance from NICE ${ }^{11}$ offers a framework for general palliative care that includes community hospitals.

When devising local strategies, primary care trusts and cancer network leads should consider these hospitals as important resources. Unlike hospices, most of which are near urban centres, community hospitals tend to serve rural communities in the less densely populated areas of the UK. Subjects for future research are the ability of community hospitals to deliver high-quality palliative care, the extent to which competing priorities and resource limitations represent barriers, and the perspectives of service users and carers on the acceptability of these hospitals as places of care near the end of life.

\section{Conclusion}

Community hospitals offer untapped potential as a means to counter inequities in access to general palliative care. Many 
already possess the resources and professional expertise, but they need to develop explicit policies within a framework for care.

Acknowledgments We thank all senior nurses and managers at the community hospitals who completed the study questionnaire and the Community Hospitals Association. This study was funded by The Health Foundation. Frances Sheldon was part of the research team but died earlier this year.

\section{REFERENCES}

1 Thomas K. Caring for the Dying at Home. Companions on a Journey. Oxford: Radcliffe, 2003

2 Addington-Hall J, McCarthy M. Dying from cancer: results of a national population based investigation. Pall Med 1995;9:295-305

3 Seale C, Cartwright A. The Year Before Death. Aldershot: Avebury, 1994

4 Higginson IJ. Who needs palliative care? J R Soc Med 1998;91:663-4

5 Field D. Special not different: general practitioners' accounts of their care of dying people. Soc Sci Med 1998;46:1111-20

6 Fordham S, Dowrick C, May C. Palliative medicine: is it really specialist territory? J R Soc Med 1998;91:568-72

7 Backhouse TW, Rich A, Fenton T. Palliative medicine: is it really specialist territory? [Letters]. J R Soc Med 1999;92:53-4

8 Buckley D, Finlay I, Fowell A, Mower MTC. Palliative medicine: is it really specialist territory? [Letters]. J R Soc Med 1999;92:100-1

9 Edmonds P, Rogers A, Shipman C. Specialist palliative care and general practice. J R Soc Med 1999;92:157

10 Thomas K. The Gold Standards Framework in community palliative care. Europ J Pall Care 2003;10:113-15

11 National Institute for Clinical Excellence. Guidance on Cancer Services. Improving Supportive and Palliative Care for Adults with Cancer. London: NICE, 2004

12 Hinton JM. Can home care maintain an acceptable quality of life for patients with terminal cancer and their relatives? Pall Med 1994; 8:183-96
13 Hanratty B. GP views on developments in palliative care services. Pall Med 2000;14:223-4

14 Munday D, Dale J, Barnett M. Out-of-hours palliative care in the UK: perspectives from general practice and specialist services. $J$ R Soc Med 2002;95:28-30

15 Bosanquet N, Salisbury C. Providing a Palliative Care Service. Oxford: Oxford University Press, 1999

16 Addington-Hall J, Higginson I. Palliative Care for Non-cancer Patients. Oxford: Oxford University Press, 2001

17 Kafetz K. What happens when elderly people die? J $R$ Soc Med 2002;95:536-8

18 Seamark D, Seamark C, Lawrence C. Contributions of community hospitals in East Devon to cancer workload. Health Trends 1997;29:114-17

19 Llewellyn J, Evans N, Walsh H. The role of community hospitals in the care of the dying. Int J Pall Nursing 1999;5:244-9

20 Seamark DA, Williams S, Hall M, et al. Palliative terminal cancer care in community hospitals and a hospice: a comparative study. $\mathrm{Br} J \mathrm{Gen}$ Pract 1998;48:1312-16

21 Seamark DA, Williams $S$, Hall $M$, et al. Dying from cancer in community hospitals or a hospice: closest lay carers' perceptions. $B r J$ Gen Pract 1998;48:1317-21

22 Seamark D, Moore B, Tucker H, Church J, Seamark C. Community Hospitals for the New Millennium. Ilminster: Community Hospitals Association, 2001

23 Grimshaw JM, Russell IT. Effect of clinical guidelines on medical practice: a systematic review of rigorous evaluations. Lancet 1993;342:1317-22

24 Burgers JS, Grol RPTM, Zaat JOM, et al. Characteristics of effective clinical guidelines for general practice. Br J Gen Pract 2003;53:15-19

25 Higginson IL, Sen-Gupta GJA. Place of care in advanced cancer: a qualitative systematic literature review of patient preferences. $J$ Pall Med 2000;3:287-300

26 Grant JA, Dowell J. A qualitative study of why general practitioners admit to community hospitals. Br J Gen Pract 2002;52:628-34

27 Department of Health. National Service Framework for Older People. London: Stationery Office, 2001

28 Department of Health. National Service Framework: Coronary Heart Disease. London: Stationery Office, 2000 